\title{
Reconstruction of leptin and dopamin molecular counter-regulation in glutamatergic hippocampal synapses
}

\author{
A.L. Proskura*, T.A. Zapara \\ Institute of Computational Technologies of SB RAS, Novosibirsk, Russia \\ *e-mail:annleop@mail.ru
}

Key words: synaptic receptors density, AMPA receptors, leptin, dopamin, dendritic spine, exciting synapses, hippocampus

Motivation and Aim: Long-term potentiation increase density of AMPARs (The $\alpha$-amino3-hydroxy-5-methyl-4-isoxazolepropionic acid receptor) at synaptic contact. In addition the receptors of various hormones, in particular leptin, growth and neurotrophic factors, as well as the receptors of other mediator systems, in particular, dopamine receptors, are present in the synaptic contacts zone. The hormone leptin crosses the blood brain barrier and regulates numerous neuronal functions, including hippocampal synaptic plasticity. The role of dopamine in the hippocampus remains poorly defined.

Results: The interactome of hippocampal dendritic spines was reconstructed (technology GeneNet was used (ROSPATENT No. 990006 from 15/02/1999)). After Longterm potentiation (LTP) a key event is the groundwork of second messenger's pools. P4 $\rightarrow$ PIP2 (PtdIns(4,5)P2) catalyzed Phosphoinositide 5-kinase (PI5K); PIP2 $\rightarrow$ PIP3 (PtdIns(3,4,5)P3) - Phosphoinositide 3-kinase (PI3K). PtdIns(4,5)P2 is hydrolyzed at inositol 1,4,5-trisphosphate (InsP3; IP3) and diacylglycerol (DAG) by phospholipase C (PLC). DAG activates protein kinase C (PKC), that phosphorylates GluR2- and GluR1AMPAR. After that GluR2-AMPARs move from and GluR1-AMPARs incorporate at the synaptic contacts. Note GluR1-AMPAR have to be phosphorylate of protein kinase A (PKA).

The activity of PKC is mediated by protein kinase PDK1 (3-phosphoinositide-dependent protein kinase 1) which binds to PtdIns $(3,4,5) \mathrm{P} 3$. Thus, PIP3 mediated a positive regulatory contour increasing the GluR1-AMPAR synaptic density: PIP2 $\rightarrow$ PIP3 $\rightarrow$ $\mathrm{PDK} 1 \rightarrow \mathrm{PKC} \rightarrow$ GluR1-AMPARs incorporation. This counteracted by PTEN, which is observed when the basic synaptic activity takes place. After LTP induction leptin promotes the removal of PTEN and, thus, support the expression of GluR1-AMPARs at the synapses. Dopamine trigger the cascade generation of CAMP that needed for activation of PKA. PKA phosphorylates of GluR1-AMPAR too that critical for anchoring them on the plasma membrane of spines and for forming the pool of AMPAR, which increases the density of synaptic AMPAR after LTP induction.

Conclusion: A model in which the synaptic contact is represented not as a passive receiver of an input signal, but as a complexly organized multicomponent molecular system has been suggested. Leptin mediates the anchoring of AMPAR at synapses. Dopamine is probably involved in the formation of a receptors pool that there may be rapid introduction to synapses after LTP induction.

Availability: http:/wwwmgs.bionet.nsc.ru/mgs/gnw/genenet/viewer/AMPA.html Acknowledgements: In issue was used data of implementation the RAS basic project of fundamental research VI 35.1.5 and RFBR grant No. 17-04-01440a. 